\title{
Total spin coherence transfer echo spectroscopy
}

\author{
J. R. Garbow, ${ }^{\text {a) }}$ D. P. Weitekamp, ${ }^{\text {b) }}$ and A. Pines \\ Department of Chemistry and Lawrence Berkeley Laboratory. University of California, Berkeley, \\ Califomia 94720 \\ (Received 19 July 1983; accepted 9 August 1983)
}

\begin{abstract}
The sensitivity of multiple quantum NMR transitions to magnetic field inhomogeneity and the relative phases and amplitudes of multiple quantum lines are discussed. The technique of total spin coherence transfer echo spectroscopy (TSCTES) is described and experimentally demonstrated. The TSCTES method allows multiple quantum spectra to be obtained which are free of inhomogeneous magnet broadening, yet remain sensitive to spin-spin couplings and chemical shift differences. The method takes advantage of the properties of the total spin coherence, the unique transition between the extreme eigenstates of a coupled spin system. Experimental results are reported for partially oriented acetaldehyde and are analyzed in terms of irreducible tensor operators. Limitations on the method and extensions to heteronuclear spin systems are also discussed.
\end{abstract}

\section{INTRODUCTION}

\section{A. Problems with existing multiple quantum methods}

The development over the last few years of time domain multiple quantum NMR has made practical the observation of all possible transitions in many systems of coupled spins. The applications of this technique have been reviewed ${ }^{1,2}$ and include the analysis of molecular structure and ordering in anisotropic phase ${ }^{3-5}$ and the characterization of spin relaxation mechanisms. ${ }^{8-8}$ Several problems arise in the practice of this method which complicate the interpretation of the spectra and limit the accuracy of the information which can be extracted.

\section{Multiple quantum spin echo experiments}

The first group of problems arises from the sensitivity of multiple quantum transitions to broadening by inhomogeneities of the magnetic field over the sample volume. Since this contribution to the linewidth is proportional to the order of the transition $n,{ }^{\circ}$ it can be resolution limiting even in situations where it is negligible in the single quantum spectrum. This has led to the incorporation of selective ${ }^{10}$ or nonselective ${ }^{11,12} \pi$ pulses at the midpoint $\left(t_{1} / 2\right)$ of the multiple quantum evolution period in order to create a spin echo at time $t_{1}$. While such an echo does effectively remove inhomogeneous broadening, it creates new difficulties.

In order to understand these difficulties, consider the Hamiltonian for a spin system consisting of $N$ coupled protons ( $I$ spins). Examples of such a system include an organic molecule in isotropic solution or dissolved in a liquid crystalline solvent, or as guest in a deuterated crystalline host. The spin Hamiltonian is

$$
\mathfrak{K}=\mathfrak{K}_{Z}+\mathfrak{H}_{D}+\mathfrak{K}_{J}
$$

The first term is the Zeeman interaction of the spins with the external magnetic field. In the resonant rotating frame, defined such that the sum of the individual chemical shifts $\omega_{i}$ is zero, it is given by

"Current address: Monsanto Company, St. Louis, Missouri 63167.

b) Current address: University of Groningen, The Netherlands.

$$
\mathcal{H}_{z}=-\sum_{i=1}^{N} \omega_{i} I_{x i}-[\omega(\mathbf{r})+\Delta \omega] I_{R},
$$

with $\int \omega(\mathbf{r}) d \mathbf{r}=0$ and $\Delta \omega$ equal to the offset from the average resonance frequency. The second and third terms in Eq. (1) are the direct and indirect couplings, respectively, among the $I$ spins

$$
\begin{aligned}
& \mathcal{H}_{D}=-\sum_{i<j} D_{i j}\left(3 I_{z i} I_{z j}-\mathbf{I}_{i} \cdot \mathbf{I}_{j}\right), \\
& \mathcal{K}_{J}=-\sum_{i<j} J_{i j} \mathbf{I}_{i} \cdot \mathbf{I}_{j} .
\end{aligned}
$$

In isotropic solutions, the direct dipolar couplings of Eq. (3a) are averaged to zero.

Figure 1 illustrates the source of the problems created by a $\pi$ pulse at $\left(t_{1} / 2\right)$. On the left, the prepared spin density operator existing during the first half of the evolution is depicted, for simplicity, by two arrows representing coherent superpositions of the connected eigenstates. After the $\pi$ pulse, one of the two pictures on the right applies, depending on whether or not

$$
\left[\mathfrak{H C}, \mathcal{H C}^{\prime}\right]=0 \text {, }
$$

where

$$
\mathfrak{H}^{\prime}=\exp \left(i \pi I_{y}\right) \mathfrak{H} \exp \left(-i \pi I_{y}\right) .
$$

$H^{\prime}$ is obtained from $\mathfrak{H C}$ by simply reversing the signs of all of the Zeeman terms.

The commutator [Eq. (4)] vanishes or is negligible when the chemical shift differences among the spins are zero or negligible compared to the resolution. The commutator also vanishes in the other extreme of weak coupling, where the chemical shift differences are much greater than the couplings between spins, so that mutual spin-flip terms may be dropped from $\mathcal{H}$. In either of these cases, Fig. 1(b) applies after the $\pi$ pulse. There is a one-to-one correspondence between coherences present before and after the $\pi$ pulse. In such cases, an average Hamiltonian ${ }^{13-15}$

$$
\mathfrak{H}_{1}=\frac{1}{2}\left(\mathfrak{H}+\mathfrak{H}^{\prime}\right)
$$

accurately accounts for the spectrum observed by Fourier transformation of the signal with respect to $t_{1}$. 

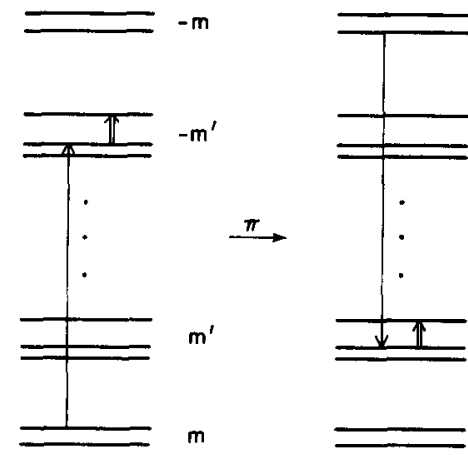

(o) Initial Coherence (b) $\left[\mathscr{H}, \mathbb{H}^{\prime}\right]=0$

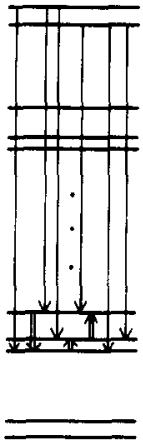

(c) $\left[\not H, \mathscr{H}^{\prime}\right] \neq 0$

FIG. 1. Coherence transfer due to a $\pi$ pulse. (a) Two coherences existing before the $\pi$ pulse are indicated by the single and double arrows. (b) When the spin Hamiltonians $\mathcal{H}$ and $\mathfrak{H}^{\prime}$ [Eq. (5)] commute, the pulse transfers each coherence to a single other coherence between states of opposite Zeeman quantum numbers. (c) When $\left[\mathcal{K}, \mathfrak{H}^{\prime}\right] \neq 0$, the situation is more complicated. Several coherences arise from each of those present before the $\pi$ pulse. This leads to a spectrum of many lines, at frequencies which are averages of those occurring before and after the $\pi$ pulse. [By permission from D. P. Weitekamp, Adv. Magn. Reson. 11, 111 (1983)].

This spectrum is free of all Zeeman terms. In liquids such a limit is referred to as $J$ spectroscopy; ${ }^{16}$ in anisotropic systems it has been used to describe multiple quantum echo spectra ${ }^{11,12}$ of sy stems with small or zero chemical shifts. If the goal is to measure spinspin couplings, this situation is satisfactory. However, the removal of inhomogeneous broadening has been gained at the expense of chemical shift information. This information may be of intrinsic interest or may at least be an aid to line assignment, particularly in liquids.

The situation is different when the commutator [Eq. (4)] is not negligible. This situation results when the chemical shift differences and spin-spin couplings are of comparable magnitude. In this case, the density operator after the $\pi$ pulse is represented by Fig. 1(c). From each coherence of order $n=\Delta M$ originally prepared, there may be a transfer to several coherences of order $-n$. The resulting spectrum cannot be characterized by an average Hamiltonian and, in fact, contains more Fourier components than would be expected from any plausible energy level diagram. The observed line positions are at the averages of pairs of those frequencies which would be observed in a perfect magnet without a $\pi$ pulse.

Such a spectrum is relatively insensitive to the chemical shift differences, though they do enter into the line positions in an attenuated form. More troublesome is that the plethora of lines created complicates spectral analysis and reduces resolution. The line intensities of such echo spectra have been calculated for single quantum spectra ${ }^{17-20}$ of some small spin systems and the extension of this calculation to multiple quantum spectra generally poses no special problems. ${ }^{19,21}$

\section{Relative amplitudes and phases of multiple quantum lines}

For simple excitation sequences, the calculation of multiple quantum spectra has been obtained analytically ${ }^{2,9,19}$ or has been programmed, ${ }^{21,22}$ but in general this is not possible. This is because the nature of the multiple quantum experiment introduces a second set of problems which are independent of any complications introduced by the $\pi$ pulse. Since the spin systems which one hopes to study using multiple quantum NMR are, in general, larger than in the single quantum experiment, corresponding calculations become lengthier. A more fundamental difficulty, though, is that the calculation requires an exact treatment of the excitation (preparation and mixing) periods, which may be not merely complex, but also to some extent unspecified, because of the unknown details of pulse imperfections. Any simulation of intensities then involves unknowns in addition to the parameters of the internal Hamiltonian. Statistical approaches have been discussed, ${ }^{22}$ but are not entirely satisfactory, giving only a qualitative picture of the overall intensity distribution. More desirable is an experiment which admits of exact and rapid calculations of relative line intensities, since these are a great aid in spectral analysis.

A final problem with multiple quantum spectra is that the line amplitudes generally are complex numbers with random phases. For this reason, the usual practice is to present magnitude spectra, which decreases somewhat the ability to resolve nearby lines.

\section{B. Coherence transfer echoes and the total spin coherence}

At the heart of our solution to these problems is the phenomenon of the coherence transfer echo. ${ }^{23-25}$ In contrast to the conventional Hahn spin echo, ${ }^{26}$ the coherence transfer echo involves a change in the magnitude of the order of coherence before and after the echo pulse. This effect has been demonstrated for a system of several protons ${ }^{25}$ and used to suppress the signal from all but one order of coherence. ${ }^{27-29}$

The method of total spin coherence transfer echo spectroscopy (TSCTES) uses this phenomenon to produce pure absorption-phase multiple quantum spectra with homogeneous linewidths for any order $n$. The line positions for a system of coupled spins are the same as would be obtained in a perfectly homogeneous magnet. The relative line intensities are calculable from the eigenvectors of the internal Hamiltonian and are independent of the excitation dynamics. Thus, this version of multiple quantum NMR eliminates all three of the aforementioned problems.

These advantages are possible due to the unique propperties of the total spin coherence, defined as the coherent superposition of the extreme eigenstates of a coupled spin system. This transition corresponds to the simultaneous flipping of all spins from down to up. Its operator may be written in bracket notation or as a product of single spin operators. For any system of $N$ spin $-\frac{1}{2}$ nuclei, these forms are 
(a)

\begin{tabular}{lllll}
\hline$U$ & $\frac{n t_{1}}{N}$ & $W$ & $t_{1}$ & $V$ \\
\hline
\end{tabular}

(b)
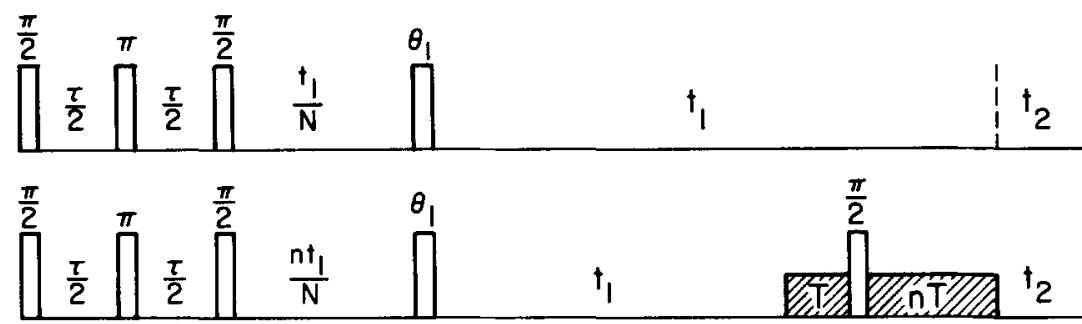

$\left.\prod^{\theta_{1}}\right\rceil_{1}$

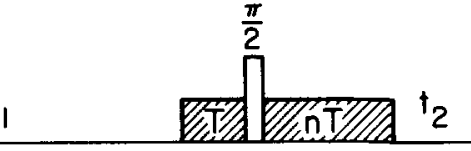

(d)

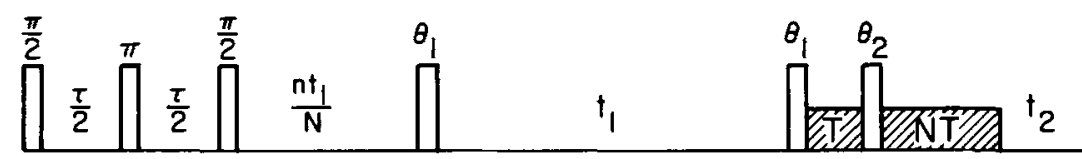

FIG. 2. (a) Generalized schematic diagram of the TSCTES experiment. The propagator $U$ excites the total spin coherence, which evolves for a time $\left(n t_{1} / N\right)$. This coherence is transferred to - $n$-quantum coherence by the pulse or sequence of pulses $W$. Free evolution of the system results in the formation of an echo at time $t_{1}$. For $n \neq 1$, a mixing period, characterized by the propagator $V$, transfers multiple quantum coherence into observable single quantum signal, which is sampled during the time period $t_{2}$. Fourier transformation with respect to $t_{1}$ gives a homogeneous $-n$-quantum spectrum. (b) Single quantum $(n=1)$ TSCTES pulse sequence. The total spin coherence is prepared by a three-pulse sequence with $\tau$ chosen by parameter proportional phase incrementation (PPPI) (Ref. 33). The propagator $V$ is omitted because single quantum coherence is directly observable. Choice of the rf nutation angle $\theta_{1}$ is discussed in Sec. IV B. (c) Simple multiple quantum TSCT ES pulse sequence. The application of a pulsed magnetic field gradient (cross-hatched areas) serves to select out only that signal resulting from coherence which is $-n$ quantum during $t_{1}$. In the present experiments, the static magnetic field homogeneity was such that no extra gradient was required. (d) Phased-line multiple quantum TSCTES pulse sequence. Coherence which is $-n$-quantum during $t_{1}$ is channeled back through the total spin coherence by the second $\theta_{1}$ pulse before mixing to single quantum. CTEF discriminates against non- $N-$ quantum coherence, while TPPI is required to separate out signals originating as different multiple quantum coherences, as described in the text.

$$
\left|\frac{N}{2}\right\rangle\left\langle-\frac{N}{2}\right|=\prod_{i=1}^{N} I_{+i}
$$

The total spin coherence is one example of a spin inversion transition. Such a transition connects eigenstates related to one another by replacing all individual spin states $|\beta\rangle$ by $|\alpha\rangle$ and $|\alpha\rangle$ by $|\beta\rangle$.

Due to the bilinear nature of spin-spin couplings, coherences between spin inversion pairs commute with $\mathfrak{H}_{D}$ and $\mathfrak{H}_{J}$ and thus evolve independently of both. ${ }^{2}$ In addition, the total spin coherence [Eq. (7)] commutes with all chemical shift differences. Thus the total spin coherence evolves independently of all terms in the spin Hamiltonian except for the inhomogeneous and homogeneous Zeeman terms in Eq. (2), which are proportional to $I_{s}$. It is this property which forms the basis of TSCTES experiments.

\section{Generalized total spin coherence transfer echo spectroscopy experiment}

A generalized schematic diagram of the TSCTES experiment is shown in Fig. 2(a). ${ }^{30}$ The propagator $U$ acts on the equilibrium density matrix $I_{x}$ to prepare total spin coherence. Although it is possible to employ selective excitation methods ${ }^{31}$ to prepare this coherence, the propagator $U$ will, in general, excite other multiple quantum orders as well. Means of suppressing that signal which does not originate as total spin coherence will be discussed below. Presently, we consider a system of $N$ spin $-\frac{1}{2}$ nuclei and focus on the evolution of the $N$-quantum coherence, whose coefficient is the density matrix element $\rho_{N / 2,-N / 2}$.

As mentioned above, the total spin coherence is only sensitive to the sum over the coupled spins of the Zeeman interaction. This $\mathrm{N}$-quantum coherence evolves freely for a time $\left(n t_{1} / N\right)$, where $-n$ is the order of the desired spectrum $(1 \leq n \leq N)$. The linear dependence of dephasing rate on multiple quantum order causes $\rho_{N / 2,-N / 2}$ to accumulate a total phase factor of $\exp \left\{+i N[\omega(\mathbf{r})+\Delta \omega]\left(n t_{1} / N\right)\right\}=\exp \left\{+i n[\omega(\mathbf{r})+\Delta \omega] t_{1}\right\}$ during this time. Following this free evolution, a transfer of coherence takes place under the action of the homogeneous propagator $W$ and the system evolves for an additional time $t_{1}$ at the frequencies of interest. For lines of order $-n$, the accumulated phase factor during $t_{1}, \exp \left\{-i n[\omega(r)+\Delta \omega] t_{1}\right\}$, just cancels the inhomogeneous dephasing and off set precession which occurred during $\left(n t_{1} / N\right)$, resulting in the formation of a coherence transfer echo. ${ }^{24,25}$ The only modulation of the amplitude of this echo is due to the chemical shift differences and spin couplings acting during $t_{1}$. Signal arising from coherence which is not $N$-quantum during time $\left(n t_{1} / N\right)$ and - $n$-quantum during $t_{1}$ decays rapidly due to the inhomogeneous Zeeman interaction $-\omega(\boldsymbol{r}) I_{\boldsymbol{s}}$.

For $n \neq 1$, the echo modulation as a function of $t_{1}$ is not directly observable and the propagator $V$ is required to mix the multiple quantum coherence back to single quantum coherence, which appears as transverse magnetization sampled during $t_{2}$. Fourier transformation of the 

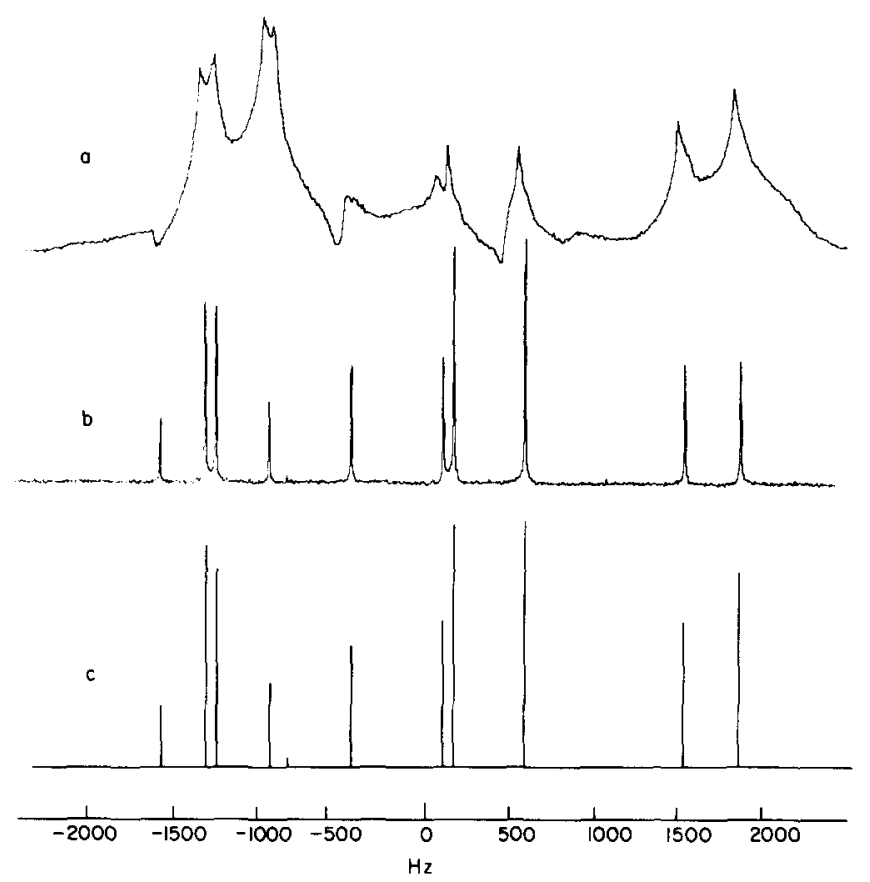

FIG. 3. Single quantum proton spectra, presented in magnitude mode, of partially oriented acetaldehyde dissolved in Eastman 15320 nematic liquid crystal. The sample used was approximately $5 \mathrm{~mm}$ in diameter and $13 \mathrm{~mm}$ long. (a) The normal single-pulse Fourier transform spectrum, showing the inhomogeneity of the magnetic field to be greater than $1 \mathrm{ppm}$. (b) The single quantum TSCTES spectrum, obtained using the pulse sequence of Fig. 2(b), is the average of three 4096-point interferograms. These were collected with $\tau=3.884 \mathrm{~ms}, \Delta t_{1}$ $=200 \mu \mathrm{s}, \theta_{1}=90^{\circ}$, and a recycle delay of $1 \mathrm{~s}$. (c) The simulation of this $A B_{3}$ system with parameters $J_{A B}=2.8 \mathrm{~Hz}, D_{A B}$ $=-179.0 \mathrm{~Hz}, D_{B B}=458.6 \mathrm{~Hz}$, and $\nu_{A B}=1360.9 \mathrm{~Hz}$. Lines of $E$ symmetry are absent because they do not correlate with the totally symmetric total spin coherence. (Reprinted with permission from Ref. 30. Copyright 1981 American Chemical Society.)

interferogram $S\left(t_{1}, t_{2}\right)$, with respect to $t_{1}$ yields the homogeneous - $n$-quantum spectrum.

\section{Outline}

In Sec. II, experimental spectra are presented for each of the pulse sequences of Fig. 2. Section III contains a density operator formalism for calculating these spectra and discusses the relative line amplitudes, including phase. In Sec. IV, the optimum line intensities for the sequence of Fig. 2(d) are related to the equilibrium magnetization. Section $\mathrm{V}$ discusses limitations on the applicability and information content of TSCTES. Section VI summarizes and mentions extensions to heteronuclear systems.

\section{SPECTRA}

\section{A. Experimental}

The experiments were performed on $\mathrm{CH}_{3} \mathrm{CHO}$ dissolved in the nematic liquid crystal $p$-octylpheny l-2chloro-4 ( $p$-heptylbenzoy loxy) benzoate (Eastman 15320). The temperature was regulated at $26.0 \pm 0.1^{\circ} \mathrm{C}$. The homebuilt spectrometer operates at $182 \mathrm{MHz}$ proton
Larmor frequency. ${ }^{32}$ The $\pi$ pulses were typically $9 \mu \mathrm{s}$ long. Sampling of transverse magnetization occurred at time $t_{2}=0$ only.

\section{B. Transfer of total spin to single quantum coherence}

The homogeneous single quantum TSCTES spectrum shown in Fig. 3(b) was obtained with the pulse sequence of Fig. 2(b). A preparation time favorable for the excitation of the total spin coherence was chosen using a recently described experimental search procedure. ${ }^{33}$ Figure 3(a) is the normal one pulse single quantum spectrum and serves to illustrate the inhomogeneity of the static magnetic field. The simulation in Fig. 3(c) confirms the sensitivity of the TSCTES spectrum to spinspin couplings and chemical shift differences.

Figure 5(a) displays the single quantum TSCTES spectrum obtained with an initial value of $t_{1}=0$. In contrast to the magnitude spectrum of Fig. 3(b), this spectrum is presented in phase sensitive mode. All lines in this spectrum are either absorptive or emissive. Both the interesting phase property and the relative intensities of these spectral lines will be discussed in Sec. IV.

\section{Multiple quantum version}

Figure 2(c) illustrates a pulse sequence used to collect multiple quantum TSCTES interferograms. The method of coherence transfer echo filtering (CTEF) $)^{27-29}$ is employed to select out only that coherence which was -n-quantum during $t_{1}$. This coherence dephases at $n$ times the single quantum rate during the time $T$ and, following a $(\pi / 2)$ mixing pulse, rephases as observable single quantum coherence. The coherence transfer echo which forms after $n T\left(t_{2}=0\right)$ is sampled. Two- and three-quantum spectra of oriented acetaldehyde obtained using this pulse sequence are displayed in Fig. 4. Within the spectral resolution $(\sim 2 \mathrm{~Hz})$, all transition frequencies in these spectra are consistent with the internal Hamiltonian parameters of Fig. 3.

Multiple quantum TSCTES spectra having phased lines of easily calculable relative intensities are obtained with the pulse sequence of Fig. 2(d). In contrast to the sequence in Fig. 2(c), coherence which spends time $t_{1}$ as $-n$ is transferred back to the total spin coherence before being mixed to observable signal. Here CTEF serves to discriminate against coherence which is not $N$-quantum during time $T$. In addition to CTEF, a second "password" is required to separate out signal which originates as total spin coherence. That addi tional discrimination is necessary is most easily seen by considering a specific example.

Assume that the spectrum of interest is the twoquantum TSCTES spectrum of a four spin system. The appropriate coherence transfer pathway may be schematically represented as

$$
4 \stackrel{\theta_{1}}{\longrightarrow}-2 \stackrel{\theta_{1}}{\longrightarrow} 4 \stackrel{\theta_{2}}{\longrightarrow}-1 \text {. }
$$

CTEF alone, however, cannot distinguish this desired pathway from the following alternate route:

$$
2 \stackrel{\theta_{1}}{\longrightarrow}-1 \stackrel{\theta_{1}}{\longrightarrow} 4 \stackrel{\theta_{2}}{\longrightarrow}-1
$$


(a)
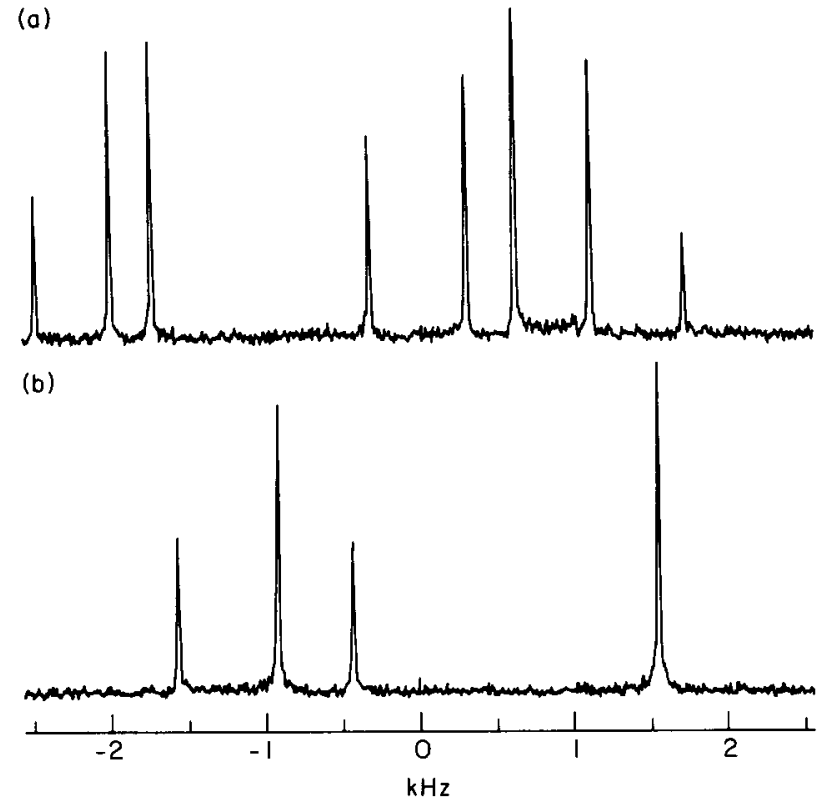

FIG. 4. Multiple quantum TSCTES spectra of partially oriented acetaldehyde using the pulse sequence of Fig. 2 (c). Each magnitude spectrum is the average of two 4096-point interferograms acquired with $\tau=3.884 \mathrm{~ms}$ and a recycle delay of $2.1 \mathrm{~s}$. (a) The two-quantum spectrum shows a total of eight lines. For this spectrum $T=9.052 \mathrm{~ms}, \Delta t_{1}=160 \mu \mathrm{s}$, and $\theta_{1}$ $=120^{\circ}$. Only a portion of the total bandwidth is displayed. (b) The three-quantum spectrum contains four lines and was collected with $T=5.564 \mathrm{~ms}, \Delta t_{1}=200 \mu \mathrm{s}$, and $\theta_{1}=139^{\circ}$.

which also leads to spectral lines that are free of inhomogeneous broadening. The characteristic modulation properties of multiple quantum coherences under phase shifts of the $\mathrm{rf}$ irradiation ${ }^{11,12,34,35}$ can serve to distinguish these two cases from one another. The phased three-quantum spectrum of Fig. 5(b) was obtained using time proportional phase incrementation (TPPI $)^{11,12}$ as the second password. This results in a clean separation of the signal contributions from the two pathways [Eqs. (8) and (9)] into different spectral regions.

\section{RELATIVE LINE AMPLITUDES}

\section{A. Tensor operators}

In the following sections on intensity and relative phase of TSCTES lines, it will prove useful to represent multiple quantum coherences as irreducible tensor operators. ${ }^{11}$ An irreducible tensor operator of rank $l$ is defined ${ }^{36}$ to be a set of $2 n+1$ operators $T_{n}^{l}(-l \leq n \leq l)$ which transform under rotation according to

$$
D(\alpha \beta \gamma) T_{n}^{l} D^{-1}(\alpha \beta \gamma)=\sum_{n^{\prime}} T_{n^{\prime}}^{l} D_{n^{\prime}, n}^{(l)}(\alpha \beta \gamma),
$$

where $D(\alpha \beta \gamma)=\exp \left(i \gamma I_{\varepsilon}\right) \exp \left(i \beta I_{y}\right) \exp \left(i \alpha I_{x}\right)$ and the $D_{n^{\prime}, n}^{(l)}(\alpha \beta \gamma)$ are elements of the Wigner rotation matrix of rank $l$. Specifically, we shall be concerned with rotations due to $\mathrm{rf}$ pulses of nutation angle $\theta$ and phase $\phi$. This allows the definition in Eq. (10) to be simplified to

$$
R(\theta, \phi) T_{n}^{l} R^{-1}(\theta, \phi)=\sum_{n^{\prime}} T_{n^{\prime}}^{l} d_{n^{l}, n}^{(l)}(\theta) \exp \left[i\left(n-n^{\prime}\right) \phi\right]
$$

with $R(\theta, \phi)=\exp \left(i \theta I_{\phi}\right)$ and $I_{\phi}=\exp \left(-i \phi I_{\varepsilon}\right) I_{y} \exp \left(i \phi I_{z}\right)$.

In all that follows it will be the magnitude of the coefficient $d_{n^{\prime}, n}^{(t)}(\theta)$ and not the phase factor $\exp \left[i\left(n-n^{\prime}\right) \phi\right]$ which is important. Both factors will be common to all lines of a given observed spectral order $n$. The
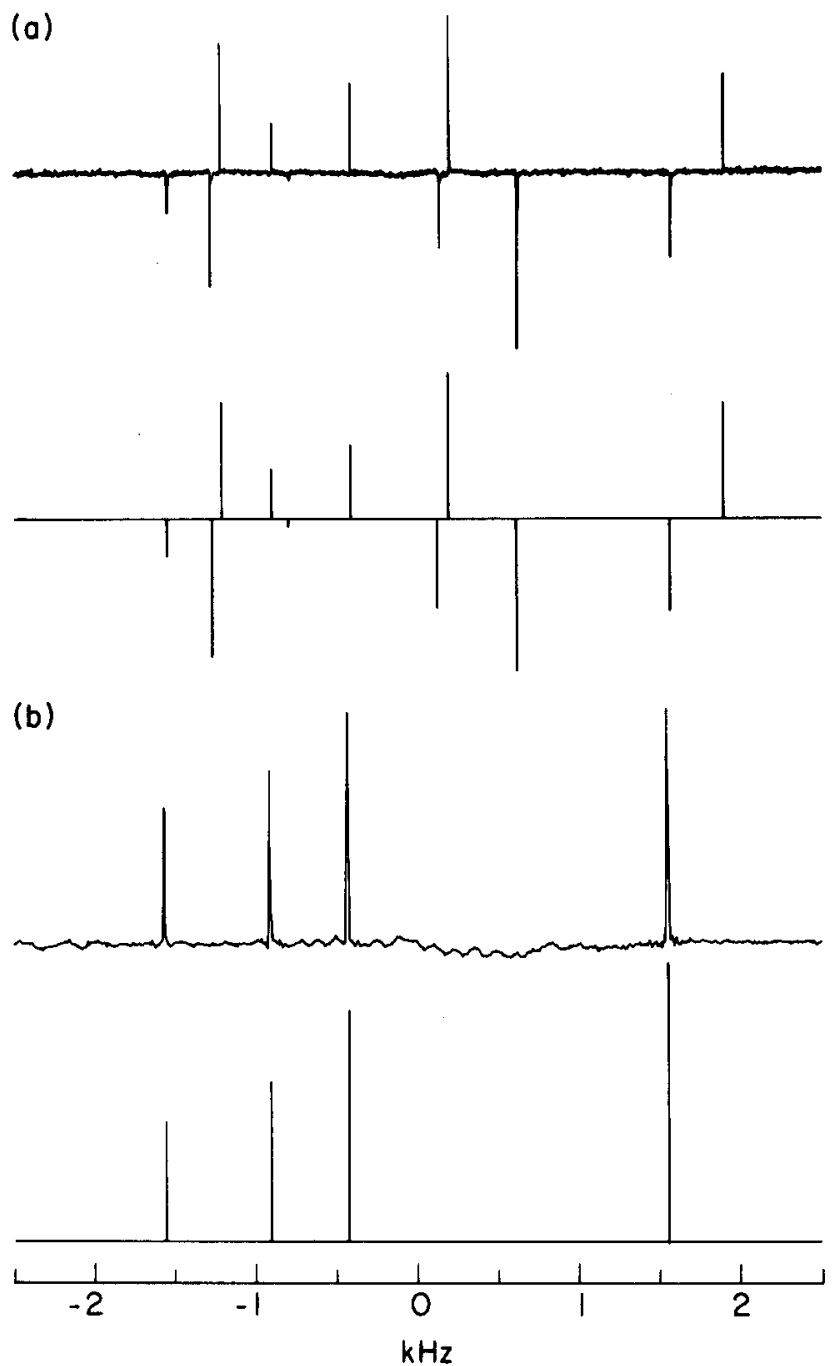

FIG. 5. TSCT ES spectra of partially oriented acetaldehyde, displayed in phase-sensitive mode, acquired with $\tau=3.884 \mathrm{~ms}$ and a recycle delay of $2.1 \mathrm{~s}$. (a) The single quantum TSCTES spectrum shows all lines as either emissive or absorptive, as discussed in Sec. III. The spectrum was collected using the pulse sequence of Fig. $2(\mathrm{~b})$ and is the average of three 4096point interferograms with $\Delta t_{1}=200 \mu \mathrm{s},\left(t_{1}\right)_{0}=0$, and $\theta_{1}=104^{\circ}$. The line positions in the simulated stick spectrum are the same as those in Fig. 3(c), the relative amplitudes being given by Eq. (19). (b) The three-quantum TSCTES spectrum collected using the pulse sequence of Fig. 2(d). TPPI was used to separate signals originating from different multiple quantum orders. This spectrum is the average of four 8192-point interferograms with $\Delta t_{1}=20 \mu \mathrm{s}, T=2.091 \mathrm{~ms}, \theta_{1}=139^{\circ}$, and $\theta_{2}$ $=104^{\circ}$. Line positions in the simulated stick spectrum were calculated using the spin-spin couplings and chemical shift difference given in Fig. 3. Relative line amplitudes are given by Eq. (21). 
former will enter into the efficiency of the experiment, while the latter can be adjusted to unity by the choice of the rf phase or by overall phase manipulation of the complex data array.

The tensor operators are normalized just as are outer products of normalized states. Thus

$$
T_{N}^{N} \equiv\left|\frac{N}{2}\right\rangle-\frac{N}{2} \mid
$$

and

$$
\operatorname{Tr}\left(T_{n}^{l} T_{-n}^{l}\right)=1
$$

\section{B. The general form of the signals}

In any NMR experiment, the measured signal is given by the trace of $\left(\rho I_{+}\right)$, where $\rho$ is the spin system density operator and $I_{+}=I_{x}+i I_{y}$ is the operator corresponding to the components of transverse magnetization. We shall first derive a general expression for the TSCTES signal based upon the schematic diagram of Fig. 2(a), and then consider the specific pulse sequences of Figs. $2(b)$ and 2(d).

$$
\begin{aligned}
& \text { We define the Hamiltonian } \mathfrak{H}_{\mathrm{INT}} \text { as } \\
& \mathfrak{H}_{\mathrm{INT}} \equiv \mathcal{H}+[\Delta \omega+\omega(\mathbf{r})] I_{\boldsymbol{z}},
\end{aligned}
$$

the Hamiltonian of Eq. (1) minus the homogeneous and inhomogeneous Zeeman interactions. It is assumed that TPPI in the preparation period and CTEF in the mixing period are used to select the coherence transfer pathway of interest. Along with the coherence transfer echo formed at $t_{1}$, the labeling of signal with these two passwords allows us to determine the $-n$-quantum TSCTES signal from the evolution under $\mathcal{F}_{\mathrm{INT}}$ of a density matrix $\rho^{(n)}(\tau)$, which is given at $t_{1}=0$ by

$$
\begin{aligned}
\rho^{(n)}(\tau) & =\rho_{N / 2,-N / 2}(\tau) \operatorname{Tr}\left(W\left|\frac{N}{2}\right\rangle\left\langle-\frac{N}{2}\right| W^{\dagger} T_{n}^{N}\right) T_{-n}^{N} \\
& =\rho_{N / 2,-N / 2}(\tau) d_{N,-n}^{(N)}\left(\theta_{1}\right) T_{-n}^{N} .
\end{aligned}
$$

In writing Eq. (15) we have assumed that $W$ is a single strong $y$ pulse. The phase is of no significant consequence here, but it will be important later that the propagator $W$ is independent of the system and can be treated as a rotation operator.

The signal at $t_{2}=0$ may then be written as

$$
S_{+}=\operatorname{Tr} \exp \left(-i \mathcal{F}_{\mathrm{INT}} t_{1}\right) \rho^{(n)}(\tau) \exp \left(i \mathcal{G}_{\mathrm{INT}} t_{1}\right) I_{+}\left(-\tau^{\prime}-T\right),
$$

where the mixing period dynamics have been incorporated into the operator

$$
I_{+}\left(-\tau^{\prime}-T\right)=V^{\dagger}\left(\tau^{\prime}, T\right)\left(I_{x}+i I_{y}\right) V\left(\tau^{\prime}, T\right) .
$$

Expanding Eq. (16) in the eigenbasis of $\mathcal{H}_{\mathrm{INT}}$ gives the signal as an interferogram in the variable $t_{1}$ :

$S_{+}=\sum_{i, j} \rho_{i j}^{(n)}(\tau)\left[x_{j i}\left(-\tau^{\prime}-T\right)+i y_{j i}\left(-\tau^{\prime}-T\right)\right] \exp \left(-i \omega_{i j} t_{1}\right)$.

The complex Fourier coefficient in front of each phase factor $\exp \left(-i \omega_{i}, t_{1}\right)$ becomes, upon Fourier transformation, the line amplitude at $\omega_{1}=-\omega_{i j}$. In the general sequence of the form of Fig. 2(a) and the particular sequence of Fig. 2(c), even the relative amplitudes depend on the mixing period. A review of this problem can be found in Ref. 2. In the following sections, we pursue the more useful situations where these relative line amplitudes are independent of both preparation and mixing dynamics.

\section{Relative single quantum line amplitudes}

For the single quantum experiment, the propagator $V$ is omitted because the -1 -quantum coherence is directly observable during $t_{1}$. Thus, $I_{+}\left(-\tau^{\prime}-T\right)$ is simply equal to $I_{+}$and the intensity of a given line is given by the product

$\rho_{i j}^{(n)}(\tau)\left(I_{+}\right)_{j i}=\rho_{N / 2,-N / 2}(\tau) d_{N,-1}^{(N)}\left(\theta_{1}\right)\left\langle i\left|T_{-1}^{N}\right| j\right\rangle\left\langle j\left|I_{+}\right| i\right\rangle$.

The first term on the right-hand side of Eq. (19) is the coefficient of the total spin coherence prepared by the propagator $U(\tau)$. The second factor is a Wigner rotation matrix element, which expresses the efficiency with which single quantum coherence is prepared from the total spin coherence by a pulse of nutation angle $\theta_{1}$. Optimal choice of this angle will be discussed in Sec. IV B. These first two factors are common to all lines. The third factor expresses the extent to which a particular transition $i-j$ is represented in the resultant single quantum operator $T_{-1}^{N}$, while the final term is the normal magnetic dipole detection period matrix element.

As demonstrated in Fig. 5(a), all of the TSCTES single quantum lines are either absorptive or emissive. In addition, the sum of the lines in this spectrum is zero. Qualitatively this is easily understood. The signal immediately following the transfer of coherence $W$ is rigorously zero, due to the orthogonality of $T_{1}^{N}$ and the detection operator $I_{+}$, which is a $T_{1}^{1}$ tensor operator component. The point $t_{1}=0$ in the interferogram therefore vanishes and, since this point is equal to the integral of the resulting spectrum, the sum of the spectral lines must be zero.

A quantitative treatment of relative line intensities and phases requires examination of the final two terms on the right-hand side of Eq. (19). The matrix elements $\left\langle j\left|I_{+}\right| i\right\rangle$ are, to within a common phase factor, all real, as are the elements $\left\langle i\left|T_{-1}^{N}\right| j\right\rangle$. Thus all of the lines have, to within a sign, a common phase. For weakly coupled systems the situation simplifies further. The sign of each matrix element alternates as the Zeeman quantum numbers $M_{i}$ and $M_{j}$ are changed by one. Thus, if the state $|i\rangle$ is taken as the initial state, the positive and negative lines originate in two orthogonal subspaces. This can be an aid in line assignment.

The simulation shown in the lower portion of Fig. 5(a) is based upon Eq. (19). The matrices describing $I_{+}$and the rotated total spin coherence were calculated in the eigenbasis of $\mathcal{H}$ with the aid of a computer. The relative intensity and phase of each line was then determined by taking the product of appropriate matrix elements. The common factor $\rho_{N / 2,-N / 2}(\tau)$ is discussed in Sec. IV C. 


\section{Relative multiple quantum line amplitudes}

We turn now to the multiple quantum TSCTES pulse sequence of Fig. 2(d). Here the mixing propagator $V$ channels $-n$-quantum coherence back through the total spin coherence before the final mixing to detectable single quantum signal. The mixing then has the same purpose as the preparation: to interconvert total spin coherence and magnetization. To emphasize this similarity we can define

$$
\rho_{-N / 2, N / 2}\left(-\tau^{\prime}-T\right)_{1} \equiv \operatorname{Tr}\left[\left|\frac{N}{2}\right\rangle\left\langle-\frac{N}{2}\right| I_{+}\left(-\tau^{\prime}-T\right)\right],
$$

and write one coefficient of Eq. (18) for the sequence of Fig. 2(d) as

$$
\begin{aligned}
\rho_{i j}^{(n)} & (\tau)\left[x_{j i}\left(-\tau^{\prime}-T\right)+i y_{j i}\left(-\tau^{\prime}-T\right)\right] \\
& =\rho_{N / 2,-N / 2}(\tau) \rho_{-N / 2, N / 2}\left(-\tau^{\prime}-T\right)\left[d_{N,-n}^{(N)}\left(\theta_{1}\right)\right]^{2}\left|\left\langle i\left|T_{-n}^{N}\right| j\right\rangle\right|^{2} .
\end{aligned}
$$

The first three factors on the right-hand side are common to all lines of the chosen order. The first is the coefficient of the total spin coherence in the prepared density matrix $\rho(\tau)$, the second the coefficient of this same coherence in the "devolved" detection operator. The last two factors are both nonnegative and real, insuring that all lines will have a common phase. The appropriate Wigner rotation matrix element comes into Eq. (21) squared, because coherence is first transferred from $N$-quantum to $-n$ and then back. For the same reason, the factor reflecting the extent to which the $i-j$ transition is represented in $T_{-n}^{N}$ enters as the magnitude squared. As in the single quantum case, the relative phases and intensities of all $-n$-quantum lines are independent of the propagators $U, V$, and $W$.

To simulate the three-quantum TSCTES spectrum of Fig. 5(b), the density matrix resulting from rotation of the total spin coherence was computer calculated. Individual line intensities were then computed by taking the magnitude squared of the appropriate elements in this density matrix.

\section{ABSOLUTE INTEGRATED INTENSITIES}

\section{A. Motivation}

In the preceding section, the relative amplitudes of TSCTES lines were discussed. We turn now to the important question of absolute spectral intensity. In Sec. IV $B$, the optimization of the pulse angles $\theta_{1}$ and $\theta_{2}$ of Fig. 2 is predicted using the well-known Wigner rotation matrices and is experimentally confirmed. In Sec. IV C, these results are combined with previous results on optimum excitation of the total spin coherence $e^{2,31}$ and on the number of lines of a given order. ${ }^{6,37}$ The results are average line intensities for sequences where Eq. (21) applies, such as that of Fig. 2(d). These intensities are expressed as a fraction of the total equilibrium magnetization and depend on both the number of spins $N$ and the order $n$ observed.

\section{B. Optimization of the pulse angles}

The dependence of the single and multiple quantum TSCTES line intensities on the pulses labeled $\theta_{1}$ in Fig. 2 are given, respectively, by Eqs. (19) and (21). In
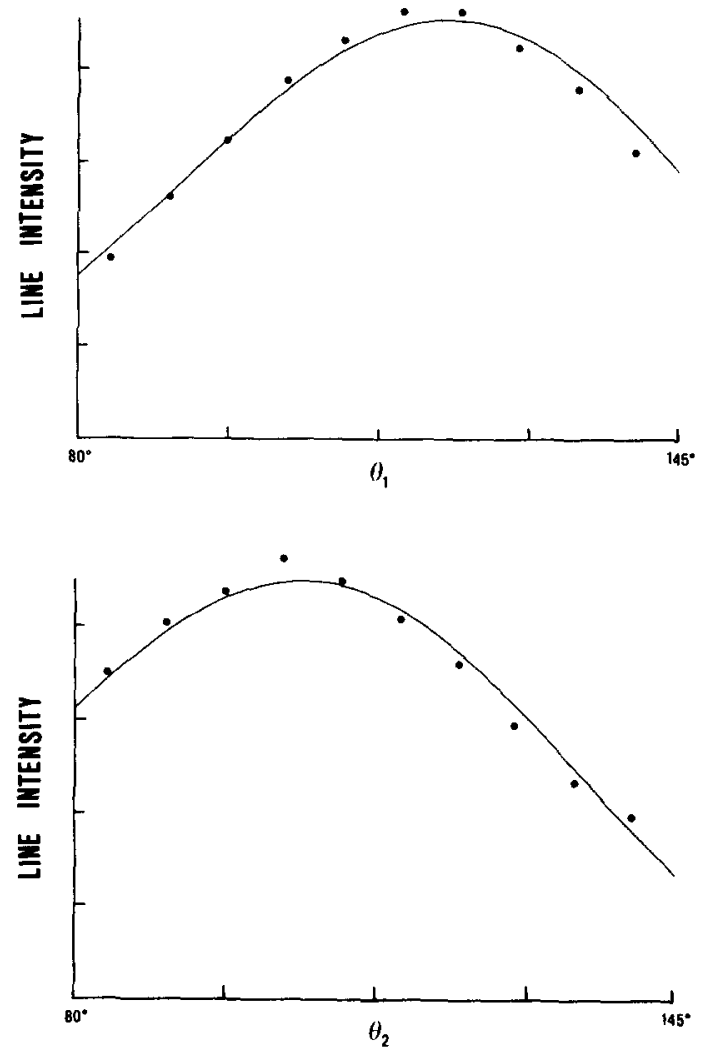

FIG. 6. Dependence of two-quantum TSCTES line intensities on the rf nutation angles $\theta_{1}$ and $\theta_{2}$ of the pulse sequence in Fig. 2 (d). Plotted line intensities are proportional to the sum of the magnitudes of the eight two-quantum lines. (a) Line intensity as a function of $\theta_{1}$, with $\theta_{2}=104^{\circ}$. The theoretical curve, shown as a solid black line, is the function $\sin ^{2} \theta_{1}\left(1-\cos \theta_{1}\right)^{2}$. (b) Line intensity as a function of $\theta_{2}$, with $\theta_{1}=120^{\circ}$. Here the theoretical curve is $\sin ^{3} \theta_{2}\left(1-\cos \theta_{2}\right)$.

addition, for the sequence of Fig. 2(d), the factor $\rho_{-N / 2, N / 2}\left(-\tau^{\prime}-T\right)$ in Eq. (21) has an implicit factor $d_{N,-1}^{(N)}\left(\theta_{2}\right)$. The relevant Wigner rotation matrix element may be found in standard texts on angular momentum ${ }^{38}$

$$
\begin{aligned}
d_{N,-n}^{(N)}(\theta)= & -1^{(N+n)}\left[\frac{(2 N) !}{(N-n) !(N+n) !}\right]^{1 / 2}\left[\cos \left(\frac{\theta}{2}\right)\right]^{N-n} \\
& \times\left[\sin \left(\frac{\theta}{2}\right)\right]^{N+n} \cdot
\end{aligned}
$$

Explicit tabulations of these coefficients have been given for $0 \leq N \leq 7^{38}$

In order to demonstrate the dependence of coherence transfer efficiency on the rf nutation angle, two sets of two-quantum 'TSCTES experiments were performed using the pulse sequence of Fig. 2(d). The first set involved varying the flip angle $\theta_{1}$ with the value of $\theta_{2}$ held constant $\left(\theta_{2}=104^{\circ}\right)$. For each value of $\theta_{1}$, the sum of the line intensities in the two-quantum spectrum was recorded. Figure 6(a) shows a plot of this total normalized spectral intensity as a function of $\theta_{1}$. The solid line is the theoretical curve, described by the function $\sin ^{2} \theta_{1}\left(1-\cos \theta_{1}\right)^{2}$. Figure $6(b)$ shows results from a second set of experiments in which flip angle $\theta_{2}$ was varied with $\theta_{1}$ constant $\left(\theta_{1}=120^{\circ}\right)$. The theoretical 


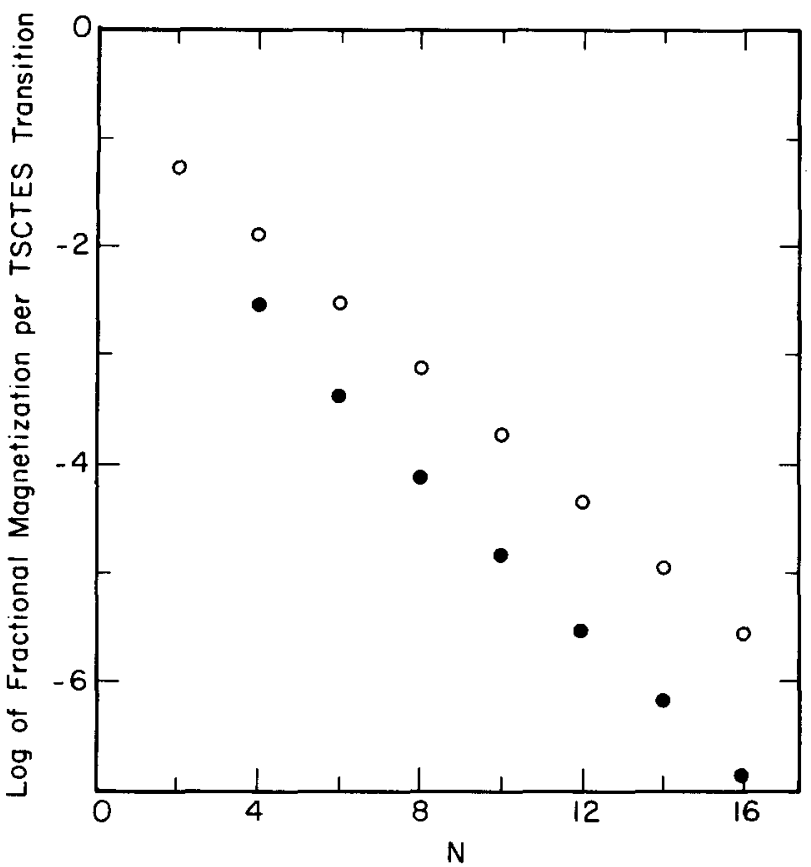

FIG. 7. The logarithm (base 10) of the mean fractional magnetization per TSCTES transition $[f(n, N) / Z(n, N)]$, as a function of the number of spins $N$ for observed orders $n=N-1$ $(O)$ and $n=N-2(\bullet)$. The calculation assumes optimum excitation periods and pulse angles for a TSCTES pulse sequence similar to Fig. 2(d). The decline in $[f(n, N) / Z(n, N)]$ with increasing $N$ is due to the greater number of $-n$-quantum transitions and the decreasing effectiveness of both exciting the total spin coherence and of transferring it to order $-n$ and back.

curve, described by the function $\sin ^{3} \theta_{2}\left(1-\cos \theta_{2}\right)$, is also shown.

The expression for $d_{N,-n}^{(N)}(\theta)$ given in Eq. (22) is easily maximized to yield optimal values of the nutation angle $\theta$ for different values of $N$ and $-n$. For the four spin acetaldehyde system $(N=4), \theta_{\text {OPT }}$ equals $104^{\circ}$ for $n=1$, $120^{\circ}$ for $n=2$, and $139^{\circ}$ for $n=3$. Each of the TSCTES spectra displayed in Figs. 4 and 5 were obtained with $\theta_{1}$ (and, when relevant, $\theta_{2}$ ) chosen according to this list.

\section{Dependence of the intensities on $N$ and $n$}

The total spectral intensity for the sequence of Fig. $2(d)$ is found by summing Eq. (21) over all lines using Eq. (13):

$$
S(n, N)=\rho_{N / 2,-N / 2}(\tau) \rho_{-N / 2, N / 2}\left(-\tau^{\prime}-T\right)\left[d_{N,-n}^{(N)}\left(\theta_{1}\right)\right]^{2} .
$$

The maximum value of $\rho_{N / 2,-N / 2}(\tau)$ for initial condition $\rho(0)=I_{g}$ and any unitary preparation propagator is $N / 2 .^{31}$ For a single channel detector, the same maximum holds for $\rho_{-N / 2, N / 2}\left(-\tau^{\prime}-T\right)$ and will be used here for the two channel TSCTES experiment as well. This is because when $N$-quantum selective sequences ${ }^{31}$ are used, the excitation is to and from a single magnetization component. Substituting these maximum values into $\mathrm{Eq}$. (23), setting $\theta_{1}=\theta_{\mathrm{OPT}}$, and dividing by the total equilibrium magnetization $\operatorname{Tr}\left(I_{z}^{2}\right)=N 2^{N} / 4$, we find the optimum fractional magnetization to be

$$
f(n, N)=N 2^{-N}\left[d_{N,-n}^{(N)}\left(\theta_{\text {OPT }}\right)\right]^{2} .
$$

Finally, it is necessary to consider that the number of lines of order $-n$ among which this magnetization will be shared also depends on $N$ and $n$. This number also depends on molecular symmetry, since only lines belonging to the totally symmetric representation enter here. An upper bound on the number of lines is given by the case of an unsymmetrical spin system where this number is ${ }^{6,37}$

$$
Z(n, N)=\left(\begin{array}{c}
2 N \\
N-n
\end{array}\right)=\frac{(2 N) !}{(N+n) !(N-n) !} .
$$

Dividing Eq. (25) into Eq. (24) gives the mean fractional magnetization per TSCTES line which can be detected in $t_{2}$. The logarithm (base 10) of this quantity is plotted in Fig. 7 for $2 \leq N \leq 16$ and $n=N-1$ and $N-2$.

\section{LIMITATIONS}

\section{A. System size, system isolation, and linewidths}

The results of Sec. IV, summarized by Fig. 7, place an obvious signal-to-noise limit on TSCTES. This is similar to that for other multiple quantum experiments. A wider discussion can be found in Ref. 2. Briefly, in the region $N \gtrsim 9$, order selective excitation ${ }^{31}$ becomes essential and the extension of even these techniques to substantially higher $N$ remains to be demonstrated.

A more subtle requirement of the TSCTES experiment is that of system isolation. If the system is only approximately limited to $N$ spins, then the $N$-quantum transition will be broadened or split by other interactions and this structure will be convoluted into all TSCTES lines.

In this regard it should be mentioned that even when all lines are resolved Lorentzians, the TSCTES linewidth (FWHM) is given by

$$
\nu_{1 / 2}^{i j}=\left[\left(\frac{n}{N}\right) \nu_{1 / 2}^{N / 2,-N / 2}+\nu_{1 / 2}^{i j}\right] \text {, }
$$

where $n=n_{i j}$ and $\nu_{1 / 2}^{i j}$ is the usual homogeneous linewidth for the $i-j^{\text {th }}$ transition. This expresses the fact that the irreversible dephasing of the total spin coherence is not removed from the dynamics by the echo.

\section{B. Diffusion}

As is the case for all echo techniques, TSCTES lines are susceptible to residual inhomogeneous broadening due to the effects of translational diffusion. ${ }^{26,40-43}$. Molecular motion from one region of magnetic field to another during the evolution period imparts a random time dependence to the inhomogeneous Zeeman interaction and thus leads to an incomplete refocusing. In such a case, Eq. (26) underestimates the linewidth.

In order to measure the effect of diffusion on linewidth, the magnetic field homogeneity was intentionally spoiled by misadjustment of the room temperature shims. Single quantum signal following a single pulse in the resultant field decayed in approximately $1 \mathrm{~ms}$. Table I shows measured one-, two-, and three-quantum TSCTES linewidths for partially oriented acetalde- 
TABLE I. Linewidths of multiple quantum TSCTES and standard multiple quantum spin echo experiments for partially oriented acetaldehyde in an inhomogeneous magnetic field.

\begin{tabular}{ccc}
\hline $\begin{array}{c}\text { Multiple quantum } \\
\text { order (n) }\end{array}$ & $\begin{array}{c}\text { TSCT ES linewidth } \\
(\mathrm{Hz})\end{array}$ & $\begin{array}{c}\text { Spin-echo } \\
\text { linewidth (Hz) }\end{array}$ \\
\hline 1 & 4 & 2 \\
2 & 5 & 3 \\
3 & 7 & 4 \\
4 & $\ldots 0$ & 5 \\
\hline
\end{tabular}

hyde in this field. Also shown are the linewidths measured in standard spin echo experiments employing a single $\pi$ pulse at the midpoint of the evolution period $\left(t_{1} / 2\right)$. TSCTES lines are seen to be more sensitive to the effects of diffusion.

This may be understood as due to the additional time period in TSCTES experiments $\left(n t_{1} / N\right)$, over which the effect of diffusion must be considered. A more quantitative treatment of diffusional effects is difficult because these experiments were not performed in the presence of a well-defined magnetic field gradient. It is apparent, however, that diffusion will not seriously limit the resolution of TSCTES experiments except in extremely inhomogeneous static fields.

\section{Information content: Symmetry and referencing}

As already noted, the lines observed with TSCTES always belong to the totally symmetric representation. This is because the total spin coherence itself has $A_{1}$ symmetry and any unitary propagator $W$ only connects this coherence with other transitions of the same symmetry. For $-(N-1)$-quantum spectra this is not a problem because all such transitions are of $A_{1}$ symmetry. For lower orders, this can be inconvenient, since transitions of other representations may be more sensitive to certain combinations of Hamiltonian parameters which one would like to extract from the spectra.

Finally, although TSCTES preserves chemical shift differences within a group of coupled spins, it is incapable of measuring differences between isolated systems. This places a limit on the technique's usefulness in mixtures and precludes the referencing of TSCTES spectra to an external standard.

\section{CONCLUSION}

The technique of total spin coherence transfer echo spectroscopy (TSCTES) has been demonstrated for both single quantum and multiple quantum spectra. Spectra which are sensitive to both chemical shift differences and spin-spin interactions between coupled protons are obtained in the absence of broadening due to the inhomogeneity of the static magnetic field. In general, multiple quantum lines are of random phase and have relative intensities which are difficult to calculate, due to the complex dynamics of the excitation periods. Experiments which first excite and then channel signal back through the unique total spin coherence produce multiple quantum spectra having phased lines and easily calculated relative intensities. The rf nutation angle dependence of coherence transfer processes has been analyzed by means of a tensor operator description and confirmed by experiment. An analy sis of the line intensities shows that TSCTES should find numerous applications in coupled spin systems of moderate size.

The extension of TSCTES to heteronuclear systems consisting of several coupled protons and $a{ }^{13} \mathrm{C}$ nucleus is straightforward. Multiple quantum spectroscopy of these systems has recently been discussed, ${ }^{29}$ including methods allowing the collection of homogeneous proton multiple quantum spectra which are sensitive to ${ }^{13} \mathrm{C}$ chemical shifts. Heteronuclear TSCTES experiments in such systems may begin with excitation of the total spin coherence of the protons or of the combined proton/ ${ }^{13} \mathrm{C}$ spin system. In a manner analogous to that described here, heteronuclear TSCTES spectra may then be collected which are sensitive to carbon-proton couplings as well as proton-proton couplings and chemical shift differences.

\section{ACKNOWLEDGMENTS}

The authors gratefully acknowledge the assistance of Jim Murdoch in the computer simulations and of Dione Carmichael in the preparation of this manuscript. This work was supported by the Director, Office of Energy Research, Office of Basic Energy Sciences, Materials Sciences Division of the U. S. Department of Energy under Contract Number DE-AC03-76SF00098.

${ }^{1}$ G. Bodenhausen, Prog. NMR Spectrosc. 14, 137 (1981).

${ }^{2}$ D. P. Weitekamp, Adv. Magn. Reson. 11, 111 (1983).

${ }^{3}$ S. Hsi, H. Zimmerman, and Z. Luz, J. Chem. Phys. 69, 4126 (1978).

${ }^{4}$ S. Sinton and A. Pines, Chem. Phys. Lett. 76, 263 (1980).

${ }^{5}$ G. Drobny, D. P. Weitekamp, and A. Pines (in preparation).

${ }^{6}$ A. Wokaun and R. R. Ernst, Mol, Phys. 36, 317 (1978).

7J. Tang and A. Pines, J. Chem. Phys. 72, 3290 (1980).

${ }^{8} \mathrm{D}$. Jaffe, R. R. Vold, and R. L. Vold, J. Magn. Reson. 46, 475 (1982), and references therein.

${ }^{9}$ W. P. Aue, E. Bartholdi, and R. R. Ernst, J. Chem. Phys. 64, 2229 (1976).

$10 \mathrm{H}$. Hatanaka and T. Hashi, J. Phys. Soc. Jpn. 39, 1139 (1975).

${ }^{11}$ G. Drobny, A. Pines, S. Sinton, D. P. Weitekamp, and D. Wemmer, Faraday Symp. Chem. Soc. 13, 49 (1979).

${ }^{12}$ G. Bodenhausen, R. L. Vold, and R. R. Vold, J. Magn. Reson. 37, 93 (1980).

${ }^{13} \mathrm{U}$. Haeberlen and J. S. Waugh, Phys. Rev. 175, 453 (1968).

${ }^{14} \mathrm{U}$. Haeberlen, High Resolution NMR in Solids, Selective

Averaging (Academic, New York, 1976).

${ }^{15}$ M. Mehring, High Resolution NMR Spectroscopy in Solids (Springer, Berlin, 1976).

${ }^{16}$ R. Freeman and H. D. W. Hill, J. Chem. Phys. 54, 301 (1971).

${ }^{17}$ A. Kumar and C. L. Khetrapal, J. Magn. Reson. 30, 137 (1978).

${ }^{18}$ A. Kumar, J. Magn. Reson. 30, 227 (1978).

${ }^{19}$ M. A. Thomas and A. Kunwar, J. Magn. Reson. 47, 535 (1982).

${ }^{20}$ D. L. Turner, J. Magn. Reson. 46, 213 (1982).

${ }^{21}$ J. B. Murdoch, Ph.D. thesis, University of California, Berkeley, 1982 (published as Lawrence Berkeley Laboratory 
Report LBL-15254).

${ }^{22}$ J. B. Murdoch, W. S. Warren, D. P. Weitekamp, and A. Pines (in preparation).

${ }^{29}$ I. Solomon, Phys. Rev. 110, 61 (1958).

${ }^{24} \mathrm{H}$. Hatanaka, T. Terao, and T. Hashi, J. Phys. Soc. Jpn. 39,835 (1975).

${ }^{25}$ A. A. Maudsley, A. Wokaun, and R. R. Ernst, Chem. Phys. Lett. 55, 9 (1978).

${ }^{26}$ E. L. Hahn, Phys. Rev. 80, 580 (1950).

${ }^{27}$ A. Bax, P. G. DeJong, A. F. Mehlkopf, and J. Smidt, Chem. Phys. Lett. 69, 567 (1980).

${ }^{28}$ Y. S. Yen and D. P. Weitekamp, J. Magn. Reson. 47, 476 (1982).

${ }^{29}$ D. P. Weitekamp, J. R. Garbow, and A. Pines, J. Chem. Phys. 77, 2870 (1982).

${ }^{30}$ D. P. Weitekamp, J. R. Garbow, J. B. Murdoch, and A. Pines, J. Am. Chem. Soc. 103, 3578 (1981).

${ }^{31}$ W. S. Warren, D. P. Weitekamp, and A. Pines, J. Chem. Phys. 73, 2084 (1980).

${ }^{32}$ J. R. Garbow, Ph.D. thesis, University of California, Berkeley, 1983 (published as Lawrence Berkeley Laboratory Report LBL-16119).
${ }^{33}$ D. P. Weitekamp, J. R. Garbow, and A. Pines, J. Magn. Reson. 46, 529 (1982).

${ }^{34} \mathrm{~S}$. Vega and A. Pines, in Magnetic Resonance and Related Phenomena, edited by H. Brunner, K. Hausser, and D. Schweitzer (Beltz-Offsetdruck, Hemsbach, West Germany, 1976), p. 395.

${ }^{35} \mathrm{~A}$. Wokaun and R. R. Ernst, Chem. Phys, Lett. 52, 407 (1977).

${ }^{36} \mathrm{~B}$. L. Silver, Irreducible Tensor Methods, An Introduction for Chemists (Academic, New York, 1976).

${ }^{37}$ R. A. Hoffman, Advan. Magn. Reson, 4, 87 (1970).

${ }^{38}$ See, for example, A. R. Edmonds, Angular Momentum in Quantum Mechanics, 2nd ed. (Princeton University, Princeton, New Jersey, 1960, p. 59.

${ }^{39}$ H. A. Buckmaster, R. Chatterjee, and Y. H. Shing, Phys. Status Solidi A 13, 9 (1972).

${ }^{40} \mathrm{H}$. Y. Carr and E. M. Purcell, Phys. Rev. 94, 630 (1954).

${ }^{41}$ E. O. Stejskal and J. E. Tanner, J. Chem. Phys. 42, 288 (1965).

${ }^{42}$ J. F. Martin, L. S. Selwyn, R. L. Vold, and R. R. Vold, J. Chem. Phys. 76, 2632 (1982).

${ }^{43}$ D. Zax and A. Pines, J. Chem. Phys, 78, 6333 (1983). 